\title{
Autoeficacia hacia la actividad física en escolares colombianos Self-efficacy towards physical activity in Colombian schoolchildren
}

*Floralinda García Puello, *Yaneth Herazo Beltrán, *Lilibeth Sánchez Guette, **Eduis Jose Barbosa Villa, ***Arelys Del Carmen Coronado Rodríguez, $* * * *$ Edy Alberto Corro Rueda, $* * * * *$ Alex Enrique Villarreal Fontalvo, $* * * * * *$ Claudia Marcela Redondo Ruíz

*Universidad Simón Bolívar (Colombia), ** Institución Educativa Santa Lucia (Colombia), *** Institución Educativa Fernando Hoyos Ripoll de Sabanalarga (Colombia), **** Institución Educativa El Concord del Municipio de Malambo (Colombia), ***** Institución Educativa Julio Pantoja Maldonado de Baranoa (Colombia), ****** Institución Educativa Normal Superior de Manatí (Colombia)

Resumen. Objetivo: El presente estudio tuvo como objetivo determinar la autoeficacia para la práctica de actividad física en escolares colombianos y establecer su relación con los niveles de actividad física identificados de acuerdo al sexo. Método: Estudio de corte transversal analítico en 2102 niñas y 2054 niños entre los 10 a 14 años estudiantes de 27 escuelas públicas. Se aplicó la Escala de Autoeficacia hacia la Actividad Física y el Cuestionario de Actividad Física para Escolares (PAQ-C) para medir el comportamiento activo. Se determinó la frecuencia de las variables categóricas y se realizó una Regresión Logística Multivariante, en la cual la variable dependiente fue la actividad física general realizada durante la última semana, considerando en el modelo como variables independientes los componentes de la autoeficacia hacia la actividad física. Se determinaron los Odds Ratio (OR) en población general y su intervalo de confianza (IC) del $95 \%$ asumiendo un valor de significancia estadística menor de 0,05 . Resultados: $64,3 \%$ de los escolares se categorizaron como inactivos físicamente, donde $66,9 \%$ de niñas resultaron inactivas y $38,4 \%$ de niños activos. Es mayor la probabilidad de ser inactivos físicamente en los escolares que consideran no poder correr al menos 8 minutos seguidos [OR 1,7 (IC95\% 1,4-1,9)], realizar actividad física después de la escuela [OR 1,5 (IC95\% 1,2-1,7)] o cuando hace mucho frio o calor [OR 1,4 (IC95\% 1,2-1,6)]. Conclusiones: Los escolares con baja autoeficacia para la práctica de actividad física tienen mayor probabilidad de ser inactivos físicamente.

Palabras clave: autoeficacia, actividad física, actividad motora, desarrollo infantil, estilo de vida saludable.

Abstract. Objective: The objective of this study was to determine the self-efficacy for the practice of physical activity in Colombian schoolchildren, and to establish their relationship with the levels of physical activity identified according to gender. Method: Analytical cross-sectional study with 2102 girls and 2054 boys between 10 to 14 years old, students from 27 public schools. The Self-Efficacy Scale was applied to Physical Activity and the Physical Activity Questionnaire for Schoolchildren (PAQ-C) to measure active behavior. Frequency of the categorical variables was determined, and a Multivariate Logistic Regression was carried out, in which the dependent variable was general physical activity carried out during the last week, whilst independent variables were the components of self-efficacy towards physical activity. The Odds Ratio (OR) in the general population and its $95 \%$ confidence interval (CI) were determined assuming a value of statistical significance of less than 0.05 . Results: $64.3 \%$ of the students were categorized as physically inactive, where $66.9 \%$ of the girls were inactive and $38.4 \%$ of the boys were active. The probability of being physically inactive is greater in schoolchildren who consider that they cannot run at least 8 minutes in a row [OR 1.7 (95\% CI 1.4-1.9)], perform physical activity after school [OR 1, 5 (IC95\% 1.2-1.7)] or when it is very cold or hot [OR 1.4 (IC95\% 1.2-1.6)]. Conclusions: Students with low self-efficacy for physical activity are more likely to be physically inactive.

Keywords: self-efficacy, physical activity, motor activity, child development, healthy lifestyle.

\section{Introducción}

La práctica regular de actividad física es considerada un factor protector para la prevención de múltiples enfermedades de tipo no transmisible, esto es corroborado por una gran variedad de estudios científicos tanto de carácter nacional como internacional (Janssen \& LeBlanc, 2010; González \& Meléndez, 2013; Prieto, Correa \& Ramírez, 2015). Sin embargo, una considerable proporción de personas, incluyendo niños y adolescentes no realiza actividad física en las intensidades recomendadas para beneficiar su salud (Hnatiuk et al, 2014; Pedro et al, 2012). En países como Canadá, Australia y China, existen prevalencias de actividad física en este grupo poblacional del $13,7 \%, 22 \%$ y $29,9 \%$ respectivamente según estudios recientes (Quan et al, 2019). En Europa, se registra que al menos el $83.2 \%$ de los niños y el $95.4 \%$ de las niñas no cumplen las recomendaciones de al menos $60 \mathrm{minu}-$ tos de actividad física semanal (De Lepeleere, 2017).

Fecha recepción: 06-08-19. Fecha de aceptación: 18-02-20

Floralinda García Puello

fgarcia@unisimonbolivar.edu.co
La Encuesta Mundial de Salud Escolar y la Encuesta de Comportamientos en Salud en la Población Escolar en el año 2010, evidenció que la prevalencia de actividad física recomendada en jóvenes de 13 a 15 años en 5 ciudades de Colombia fue del 15\% (Piñeros \& Pardo, 2010), prevalencia que aumenta al $26 \%$ en el año 2016 con mejores niveles en hombres en comparación con las mujeres (27.62 vs.4.2\%) según el Report Card on Physical Activity for Children and Youth (González et al, 2016). Por otro lado, un estudio realizado en escolares de la ciudad de Bogotá, evidenció prevalencias de actividad física del $42,3 \%$ y $31,0 \%$ en hombres y mujeres en etapa escolar respectivamente (Prieto, Correa \& Ramírez, 2015).

Existe suficiente evidencia de la disminución en la práctica regular de actividad física en niños en transición a la etapa adolescente (Méndez, Cecchini \& Fernández, 2017), lo cual, sumado a hábitos inadecuados de alimentación, y niveles elevados de ocio digital sedentario (conectividad a internet, videojuegos, teléfono móvil y televisión), son considerados factores clave para el padecimiento de todo tipo de enfermedades (Núñez et al, 2019), (Ríos et al, 2018) donde el sobrepeso, la obesidad, asociadas a su vez con hipertensión, diabetes tipo 2, enfermedad cardiovascular y algunos tipos 
de cáncer ocupan los primeros lugares en las estadísticas de mortalidad prematura en la población (Seguin et al, 2019).

Son múltiples las publicaciones científicas que evidencian el impacto positivo que la actividad física tiene sobre el crecimiento y desarrollo de los niños en etapa escolar, así como las oportunidades que brinda para su participación en diferentes contextos de recreación, cultura y ocio (Chanal et al, 2019; Rosa, García y Carrillo, 2019). De igual forma, está suficientemente reportado el impacto positivo que la actividad física en la niñez tiene sobre el nivel de actividad física en la edad adulta y la relación inversa entre esta y el aumento de la grasa corporal, la cual se asocia positivamente con la enfermedad cardiovascular y de forma negativa con la depresión (Denton et al, 2013).

Desafortunadamente, es también creciente el número de estudios que muestra una tendencia a la disminución progresiva del nivel de actividad física en este grupo poblacional, al tiempo que se informa de un aumento en los problemas de salud general (Hui-ping Sit et al, 2019). Los malos hábitos alimenticios y el uso exacerbado de la tecnología son algunos de los factores más frecuentemente identificados como responsables de la inactividad física en este grupo etario. Estos factores se relacionan con los modos de vida contemporánea y se presentan tanto en el ámbito familiar como en el escolar (Popeska et al, 2018).

Estos datos señalan la necesidad de realizar intervenciones para mejorar las conductas saludables de los niños en etapa escolar, específicamente las encaminadas a la promoción de la práctica de actividad física regular. Para esto, resulta fundamental conocer aspectos relacionados con las creencias y comportamientos de este grupo poblacional y aplicarlos al estudio de la actividad física. La Teoría Social Cognitiva, específicamente la autoeficacia, juega un papel fundamental en este campo dado que explica como las creencias que tienen los individuos acerca de su capacidad para realizar determinadas acciones puede llevarlos al logro de metas positivas en situaciones específicas (Kitzman et al, 2010), en otras palabras, esta teoría plantea la capacidad y/o competencia que tiene un individuo para hacerle frente de manera satisfactoria a situaciones y retos de la vida (Hernández et al, 2011).

La autoeficacia puede funcionar como un indicador de adherencia a los programas de actividad física, así como de la práctica de acciones y comportamientos relacionados con estilos de vida saludables en las poblaciones (Aedo \& Ávila, 2009). Las personas que presentan elevados niveles de autoeficacia son las que normalmente se comprometen más con el cumplimiento de las recomendaciones para la práctica de actividad física de forma regular, además esta puede ser considerada un indicador del tiempo e intensidad con la que las personas pueden comprometerse en la realización de ciertas actividades y de su rendimiento (Jáuregui et al, 2013).

El presente estudio tuvo como objetivo determinar la autoeficacia para la práctica de actividad física en escolares y establecer su relación con los niveles de actividad física identificados.

\section{Material y método}

Estudio de corte transversal analítico en 2102 niñas y
2054 niños entre los 10 a 14 años estudiantes de 27 escuelas públicas. De una población total de 19933 escolares se seleccionó la muestra de 4156, teniendo en cuenta un nivel de confianza del $95 \%$ y un poder del $80 \%$. La población de estudiantes fue seleccionada de manera probabilística y aleatoria, se escogió proporcional al número de escolares en cada curso, de tercero a noveno grado; y para obtener una representatividad según el sexo de los sujetos, se decidió escoger el $51 \%$ de niñas y el $49 \%$ de varones. La escuela proporcionó el listado académico suministrado facilitando la selección aleatoria. Una vez los directivos de cada escuela aprobaron la realización del estudio, se solicitó el consentimiento de los padres de familia.

Cada niño durante su jornada escolar (durante el recreo o en clases) respondió las preguntas contenidas en la Escala de Autoeficacia hacia la Actividad Física, desarrollada por Aedo et al, (Aedo \& Ávila, 2009) mostrando una consistencia alfa de Cronbach de 0,733 y confiabilidad test-retest de 0,867. La Escala utiliza una escala dicotómica (sí o no) y contiene 12 ítems o componentes: No creo que puedo hacer actividad física, ejercicio o deporte después de la escuela porque también veo TV o juego videojuegos, después de la escuela porque mis amigos quieren que haga alguna otra cosa, correr al menos 8 minutos sin parar, porque hace calor o frío afuera, porque me siento cansado, porque tengo mucha tarea, porque me quedo en casa, porque mis amigos creen lo contrario y porque tengo otras clases en las tardes; asimismo, incluye dos ítems adicionales: no creo que tengo la habilidad necesaria para jugar el deporte que quiera o para hacer ejercicio y que algunos de mis padres puedan llevarme a practicar deporte o hacer ejercicio en la tarde.

También, se utilizó el Cuestionario de Actividad Física para Escolares (PAQ-C) para medir el comportamiento activo. El PAQ-C contiene 9 preguntas sobre las actividades realizadas durante el tiempo libre, clases de educación física, recreo, almuerzo, después de la escuela, en las tardes y los fines de semana; permite calcular los niveles de actividad física a través del promedio de las respuestas del 1 al 5 de las 9 preguntas, donde los estudiantes por encima del promedio 2,73 se consideraron activos. El PAQ-C en niños colombianos mostró una consistencia interna de 0,73 en la primera medición y 0,78 en la segunda, y un Coeficiente de Correlación Intraclase de 0,60 (Herazo \& Domínguez, 2012). Se indagó sobre las características sociodemográficas sexo, edad y estrato socioeconómico.

Para el análisis de los datos se utilizó el programa estadístico SPSS versión 24, licencia Universidad Simón Bolívar. Se estableció la frecuencia de las variables categóricas y para determinar la influencia de las actividades realizadas en cada momento del día y de la semana en los niveles activo o inactivo se realizó una Regresión Logística Multivariante, en la cual la variable dependiente fue la actividad física general realizada durante la última semana, considerando en el modelo como variables independientes los componentes de la autoeficacia hacia la actividad física. Se determinaron los Odds Ratio (OR) en población general y su intervalo de confianza (IC) del $95 \%$. Se asumió un valor de significancia estadística menor de 0,05 . Los OR general se ajustaron por sexo y edad. 


\section{Resultados}

Se observan en la Tabla 1 las principales características de los niños y niñas participantes. Todos los escolares pertenecen a un estrato socioeconómico bajo. Una mayor participación de niñas $(50,6 \%)$ y de $6^{\circ}$ grado escolar $(34,8 \%)$. La media de edad fue $12 \pm 1,3$ años.

Los niveles de actividad física se presentan en la Tabla 2. El 64,3\% de los escolares se categorizaron como inactivos físicamente; al analizar la prevalencia de actividad física según sexo, se encontraron diferencias significativas, más niñas inactivas $(66,9 \%)$ y niños activos $(38,4 \%)$.

La Tabla 3 muestra la proporción de estudiantes que perciben no poder realizar actividad física ante las diferentes situaciones planteadas. Se resalta en población general, que el $72 \%$ tiene la creencia que sus padres no los llevarán a

$$
\text { Tabla } 1 .
$$

\begin{tabular}{|c|c|c|}
\hline Sexo & Frecuencia & Porcentaje \\
\hline Femenino & 2102 & 50,6 \\
\hline Masculino & 2054 & 49,4 \\
\hline \multicolumn{3}{|c|}{ Grado escolar } \\
\hline $3^{\circ}$ grado & 18 & 0,4 \\
\hline $4^{\circ}$ grado & 256 & 6,2 \\
\hline $5^{\circ}$ grado & 429 & 10,3 \\
\hline $6^{\circ}$ grado & 1448 & 34,8 \\
\hline $7^{\circ}$ grado & 908 & 21,8 \\
\hline $8^{\circ}$ grado & 635 & 15,3 \\
\hline $9^{\circ}$ grado & 462 & 11,1 \\
\hline \multicolumn{3}{|l|}{ Edad } \\
\hline 10 años & 754 & 18,1 \\
\hline 11 años & 801 & 19,3 \\
\hline 12 años & 877 & 21,1 \\
\hline 13 años & 865 & 20,8 \\
\hline 14 años & 859 & 20,7 \\
\hline
\end{tabular}

$$
\text { Tabla } 2 .
$$

Niveles de actividad física en los estudiantes participantes

\begin{tabular}{lcccl}
\multicolumn{5}{l}{ Niveles de actividad física en los estudiantes participantes } \\
\hline Inactivos & $2672(64,3 \%)$ & $1407(66,9 \%)$ & $1265(61,6 \%)$ & 0,0001 \\
Activos & $1484(35,7 \%)$ & $695(33,1 \%)$ & $789(38,4 \%)$ & \\
\hline
\end{tabular}

Tabla 3.

Autoeficacia para la actividad física en población general y según el sexo de los escolares

$\frac{\text { Autoeficacia para la actividad fisica en poblacion general y según el sexo de los escolares }}{\text { Frecuencia (Porcentaje) }}$ \begin{tabular}{lccc} 
No creo que puedo: & $\begin{array}{c}\text { goblación } \\
\text { general }\end{array}$ & & Niñas \\
\hline $\begin{array}{l}\text { Hacer actividad física después de la escuela la } \\
\text { mayoría de los días entre semana }\end{array}$ & $967(23,3)$ & $539(55,7 \%)^{*}$ & $428(44,3 \%)^{*}$
\end{tabular} $\begin{array}{llll}\text { Hacer actividad física después de la escuela } & 1324(31,9 \%) & 737(55,7 \%)^{*} & 587(44,3 \%)^{*}\end{array}$

porque también veo TV o juego videojuegos porque mis amigos quieren que haga alguna otra

por

$\begin{array}{llll}\text { Cosa } & 1481(35,6 \%) & 807(54,5 \%)^{*} & 674(45,5 \%)^{*}\end{array}$ $\begin{array}{llll}\text { Correr al menos } 8 \text { minutos sin parar } & 1481(35,6 \%) & 807(54,5 \%)^{*} & 674(45,5 \%)^{*} \\ \text { Hacer actividad fisica porque hace calor o frío } & 1530(36,8 \%) & 875(57,2 \%)^{*} & 655(42,8 \%)^{*}\end{array}$ Hacer actividad fisica porque hace calor o frio $1530(36,8 \%) \quad 875(57,2 \%)^{*} \quad 655(42,8 \%)$
afuera

$\begin{array}{llll}\text { Hacer ejercicio porque me siento cansado } \quad 2337(56,2 \%) & 1277(54,6 \%)^{*} \quad 1060(45,4 \%)\end{array}$ Hacer actividad fisica porque tengo mucha tarea $2473(59,5 \%) 1293(52,3 \%)^{* *} 1180(47,7 \%)^{*}$ $\begin{array}{lll}\text { Hacer actividad fisica porque me quedo en casa } 1079(26 \%) & 509(47,2 \%)^{* *} \quad 570(52,8 \%)^{*}\end{array}$ $\begin{array}{lrrr}\text { Hacer ejercicio o algún deporte porque mis } & 1308(31,5 \%) & 671(51,3 \%) & 637(48,7 \%)\end{array}$ amigos creen lo contrario

$\begin{array}{lll}\text { Hacer actividad física porque tengo otras clases } 2288(55,1 \%) & 1224(53,5 \%)^{*} \quad 1064(46,5 \%)\end{array}$ Hacer activi
en las tardes

$\begin{array}{llll}\text { No las tardes } & & \end{array}$ que tengo la habilidad necesaria para $\quad 2674(64,3 \%) \quad 1324(49,5 \%) \quad 1350(50,5 \%)$

jugar el deporte que quiera o para hacer

ejercicio

No creo que algunos de mis padres puedan $\quad 2994(72 \%) \quad 1506(50,3 \%) \quad 1488(49,7 \%)$ llevarme a practicar deporte o hacer ejercicio e

la tarde

${ }^{*} \mathrm{p}<0,0001{ }^{* *} \mathrm{p}<0,05$

Tabla 4

Relación entre los componentes de la autoeficacia hacia la práctica de actividad física y los niveles de actividad física ajustada por sexo

\begin{tabular}{lll}
\hline Componentes de la autoeficacia hacia la actividad física & OR & IC $95 \%$
\end{tabular} No creo que puedo:

\begin{tabular}{lll}
\hline Hacer actividad física después de la escuela la mayoría de los días entre semana & 1,5 & $1,2-1,7$
\end{tabular} Hacer actividad física después de la escuela porque también veo TV o juego 1,1 0,9-1,2 videojuegos

Hacer ejercicio o deporte después de la escuela porque mis amigos quieren que $\quad 1,4 \quad 1,2-1,6$ haga alguna otra cosa

Correr al menos 8 minutos sin parar

Hacer actividad física porque hace calor o frío afuera

Hacer ejercicio porque me siento cansado

Hacer actividad física porque tengo mucha tare

Hacer actividad fisica porque me quedo en casa

Hacer ejercicio o algún deporte porque mis amigos creen lo contrario

Hacer actividad física porque tengo otras clases en las tardes

$\begin{array}{lll}\text { Hacer actividad física porque tengo otras clases en las tardes } & 1,1 & 0,9-1,2 \\ \text { No creo que tengo la habilidad necesaria para jugar el deporte que quiera o para } & 0,8 & 0,7-1,1 \\ \text { hacer ejercicio } & \end{array}$

No creo que algunos de mis padres puedan llevarme a practicar deporte o hacer $\quad 1,2 \quad 0,95-1,4$ ejercicio en la tarde practicar actividad física en la tarde después de la escuela; un porcentaje también alto, $64,3 \%$, percibe que no tienen la habilidad para jugar su deporte preferido. Al comparar entre niños y niñas, se encuentran diferencias significativas en las creencias y percepciones sobre la realización de deporte y ejercicio según el sexo; más niñas $(57,2 \%)$ opinando que no realizan actividad física porque hace calor o frío en los espacios externos, en comparación de los niños $(42,8 \%)$.

$\mathrm{Al}$ analizar la relación entre los componentes de la autoeficacia hacia la práctica de actividad física y los niveles de actividad física se encontró que es mayor la probabilidad de ser inactivos físicamente en los escolares que consideran no poder correr al menos 8 minutos seguidos [OR 1,7 (IC95\% $1,4-1,9)]$, realizar actividad física después de la escuela [OR 1,5 (IC95\% 1,2-1,7)] o cuando hace mucho frio o calor [OR 1,4 (IC95\% 1,2-1,6)] (Tabla 4)

\section{Discusión}

Los resultados obtenidos en relación con los niveles de actividad física en nuestro estudio evidencian que más del $64 \%$ de los escolares son inactivos, dato que resulta similar al encontrado en otro estudio realizado en la ciudad de Barranquilla en una muestra de 991 niños de 17 escuelas públicas, donde el $65,4 \%$ fueron categorizados con niveles bajos de actividad física; es decir, que no cumplían con las recomendaciones de práctica semanal establecidas por la Organización Mundial de la Salud (Campo et al, 2017). Es importante resaltar que la magnitud de inactividad física existente en los estudios anteriores se presenta en el estrato socioeconómico bajo, relacionado a su vez con la pertenencia a escuelas públicas, variables que fueron relacionadas por Bauman et al. (2012), quien reportó en su estudio la asociación existente entre variables sociodemográficas y actividad física, destacando mayor prevalencia de niveles más bajos en los países de mediano y bajo ingreso en contraste con los resultados obtenidos en los países de ingresos altos, donde se evidenciaron mejores niveles de actividad física en ambos sexos y los diferentes grupos etarios. Por su lado, Briceño et al. (2018), encontraron en su estudio una prevalencia de inactividad física del $85,6 \%$, en el cual compara escolares de áreas rural y urbana en Colombia, encontrando peores resultados en el área urbana.

La asociación existente entre inactividad física y estrato socioeconómico bajo es una constante en los principales países de América Latina, este fenómeno guarda estrecha relación con los efectos de la globalización sobre los estilos de vida, donde la industrialización, caracterizada por comportamientos sedentarios e inadecuados hábitos alimenticios afectan principalmente a los grupos de edad entre 5 y 17 años (González \& Martner, 2013; Encuesta Nacional de la Situación Nutricional en Colombia. 2010; Rivera et al, 2013).

En general, según declaraciones de la Organización Mundial de la Salud(OMS) y la Organización Panamericana de la Salud (OPS) en América Latina y el Caribe existe una elevada prevalencia de Enfermedades no transmisibles, lo que las convierte en una amenaza importante para la salud pública, estas enfermedades se encuentran asociadas a comportamientos poco saludables, donde la inactividad física ocupa uno de los primeros lugares junto con el hábito de 
fumar y la alimentación inadecuada (Alemán et al, 2018). La mayor parte de los países de América latina presentan datos preocupantes sobre la frecuencia de práctica de actividad física de sus poblaciones. Uruguay declaró en el año 2012 que menos de un tercio de sus adolescentes (28.8\%) cumplió con las recomendaciones de 60 minutos diarios de actividad física, evidenciando niveles más bajos en las niñas (González \& Martner, 2013). En Chile, un estudio identificó una prevalencia de nivel bajo de actividad física del $39,6 \%$ en una muestra de 443 niños entre los 6 y 13 años. Sin embargo, se aclara que estos últimos resultados se obtuvieron a través de la aplicación de otra escala de medición (Álvarez et al, 2016).

En otros contextos culturales, los resultados de las mediciones de actividad física a través del cuestionario PAC - C muestran resultados diversos, en España por ejemplo, se han encontrado frecuencias de inactividad física en este grupo poblacional del 27,8\%, 32,9\% y 33\% (Guillamón et al, 2019; Manchola, Bagur \& Girabent, 2017; Arriscado et al, 2015). Estos hallazgos difieren de los obtenidos en un grupo de escolares japoneses donde la prevalencia de inactividad física fue del 36,4\% (Isa et al, 2019) evidenciando una frecuencia elevada de estudiantes activos (63,6\%). Otros estudios también reportan mayor frecuencia de niveles adecuados de actividad física entre la población de niños escolares afroamericanos, europeos, e hispanos (Erdim, Ergun \& Kuðuoðlu, 2019; Moore et al, 2019).

En relación con la prevalencia de actividad física según sexo, el presente estudio encontró diferencias significativas, siendo las niñas más inactivas $(66,9 \%)$ que los niños $(38,4 \%)$, esto resulta consistente con estudios previos; muestra de esto fue lo hallado por Isa $\mathrm{T}$, donde se evidencia que la puntuación media de la versión japonesa del PAQ-C fue significativamente más alta en hombres en comparación con las mujeres (Isa et al, 2019), asimismo, Sallis (1993) dio cuenta de estas diferencias enfatizando a través de su estudio que los hombres eran más activos que las mujeres. En Colombia, un estudio encontró resultados similares por sexo, además de diferencias significativas en relación con variables antropométricas y condición física donde las niñas presentaron peores calificaciones (Vidarte, Vélez \& Parra, 2019). Sin embargo, otro estudio nacional arrojó valores más elevados de actividad física tanto vigorosa como moderada en escolares de sexo femenino, los niños sólo obtuvieron niveles superiores a las niñas en la categoría de caminata (Palomino, Reyes \& Sánchez, 2018). La variabilidad existente entre los diversos estudios insta a la comunidad científica a seguir desarrollando investigaciones que evalúen de forma continua la variable de actividad física en este grupo poblacional, de tal forma que el diseño de las intervenciones se adecúe cada vez más a las necesidades y requerimientos de cada grupo en particular en relación con las diferencias existentes entre los sexos.

Con respecto a las variables de autoeficacia, el presente estudio evidenció que un $72 \%$ de los escolares tiene la creencia de no poder realizar actividad física después de la escuela por no contar con el apoyo de sus padres; este resultado es similar al obtenido por Olaya, Bastidas y Arvidsson (2015), donde la frecuencia en la práctica de actividad física en esta población se asoció con el apoyo social y la relación con los padres. Las actividades realizadas conjuntamente entre padres e hijos han sido evidenciadas como un factor importante para el desarrollo de estilos de vida saludables tanto en niños y adolescentes (Castillo, Tornero y García 2018). Asimismo, diversos estudios han corroborado que los niños y adolescentes tienen mayor probabilidad de ser activos físicamente cuando sus familiares participan en actividades físico/recreativas y deportivas de forma regular, los impulsan a practicarlas, y lo hacen juntos (Fradejas \& Espada, 2018).

Asimismo, nuestros resultados muestran que un $64,3 \%$ de escolares percibe que no tiene las habilidades necesarias para jugar su deporte favorito. Este aspecto, relacionado con la autopercepción de capacidades físicas y competencias motrices resulta común entre los escolares con niveles pobres de actividad física (Manley et al, 2014).

En general, esta investigación arrojó diferencias significativas en las creencias y percepciones sobre la realización de deporte y actividad física en los escolares según sexo, siendo las niñas las que en mayor proporción $(57,2 \%)$ expresan que no realizan actividad física por diversos aspectos personales y externos relacionados con la temperatura ambiental (calor o frío). Estos resultados son comparables a los obtenidos por Fairclough, Ridgers \& Welk (2012), quien evidencia que en general las niñas son menos activas que los niños, tienen menor capacidad física percibida y mayor insatisfacción corporal, para este autor, la competencia percibida para la práctica de actividad física puede estar relacionada con el sexo, debido precisamente a que los niños tienen mejores niveles de actividad física y se autoperciben con mayor fuerza y competencia deportiva que las niñas (Crespo eta al, 2013). De igual forma, Carissimi et al. (2017) encontró en su estudio que la percepción de las habilidades físicas fue mayor en los niños que en las niñas, reforzando así este criterio sustentado por múltiples investigaciones.

En síntesis, este estudio encontró que los escolares con baja autoeficacia para la práctica de actividad física tienen mayor probabilidad de ser inactivos físicamente. Este resultado es consistente con lo obtenido por Suton et al. (2013) en su investigación, donde se evidencia que los escolares que presentan puntuaciones altas de autoeficacia física tienden a participar de forma significativamente mayor en actividades físico recreativas que los niños con puntuaciones bajas de autoeficacia física.

Son múltiples los estudios que han investigado la relación entre la autoeficacia y la actividad física, concluyendo que el desarrollo temprano de actitudes positivas hacia esta práctica tiene un papel importante sobre la tendencia personal hacia la adquisición de un estilo de vida activo y saludable en edades posteriores (Biddle \&Goudas, 1996; Zurita et al,2017).

\section{Conclusiones}

Los resultados del presente estudio permiten concluir que la autoeficacia es un indicador válido para predecir la práctica de actividad física en niños. Asimismo, es una herramienta valiosa para determinar el éxito que se tendrá al llevar a cabo dicha práctica, determinado en gran medida por la confianza que la persona siente en sí misma al momento de realizar o mantener esa conducta. Esta convicción influirá en 
cómo el niño le hará frente a los retos que plantea la práctica de actividad física, en el grado de esfuerzo que emplee y en la perseverancia mantenida frente a los mismos (Aedo \&Ávila, 2009; Biddle \&Goudas, 1996).

Con base en todo lo expuesto, son claras las repercusiones prácticas que el presente estudio tiene en el campo de la salud pública, específicamente en la línea de promoción de la salud, puesto que fundamenta a través del modelo de la autoeficacia el diseño de intervenciones encaminadas al aumento de hábitos físico-saludables en la población escolar, especialmente de la práctica regular de actividad física como un elemento asociado positivamente con la salud física, mental y social de este grupo poblacional en particular (Zurita et al, 2017); estas intervenciones deben enfocarse en las particularidades y diferencias existentes entre niños y niñas percibidas por diversos autores en múltiples investigaciones, tomando con referente fundamental para el diseño de dichos programas la variable de autoeficacia. Asimismo, el análisis minucioso de los resultados de la presente investigación, permitirán orientar nuevas investigaciones sobre la influencia de factores de tipo psicosocial en la práctica de actividad física en la edad escolar, más aún reconociendo que sigue siendo insuficiente la evidencia científica sobre el estudio de los factores predictores de la AF de niños y adolescentes (Martínez, Mayorga \& Viciana, 2018).

\section{Agradecimientos}

Los autores agradecen el apoyo científico y logístico de la Vicerrectoría de Investigación e Innovación de la Universidad Simón Bolívar para desarrollo del presente estudio.

\section{Referencias}

Aedo, Á., Ávila, H. (2009). Nuevo cuestionario para evaluar la autoeficacia hacia la actividad física en niños. Rev Panam Salud Publica, 26(4), 324-329.

Aleman, A., Colistro, V., Colomar, M., Cavalleri, F., Alegretti, M., Bugñioli, M. (2018). Carga de enfermedad de los adolescentes en Uruguay y su comparación con la de América Latina y el Caribe. Ciência \& Saúde Coletiva, 23(9), 2813-2820.

Álvarez, C., Ramírez, R., Martínez, C., Vallejos, A., Jaramillo, J., Salas, C., Cano, J., Celis, C. (2016). Hipertensión en relación con estado nutricional, actividad física y etnicidad en niños chilenos entre 6 y 13 años de edad. Nutr Hosp, 33:220-225. DOI: $10.20960 /$ nh.93

Arriscado, D., Muros, J., Zabala, M., Dalmau, J. (2015). Hábitos de práctica física en escolares: factores influyentes y relaciones con la condición física. Rev Nutr Hosp, 31, 1232-1239. DOI:10.3305/nh.2015.31.3.8186.

Bauman, A., Reis, R., Sallis, J., Wells, J., Loos, R., Martin, B., for the Lancet Physical Activity Series Working Group. (2012). Correlates of physical activity: why are some people physically active and others not?. Lancet, 380, 258-71. doi.org/10.1016/ S0140-6736(12)60735-1

Biddle, S., Goudas, M. (1996). Analysis of Children's Physical Activity and its Association with Adult Encouragement and Social Cognitive Variables. J Sch Health, 66(2), 75-84. doi.org/ 10.1111/j.1746-1561.1996.tb07914.x

Briceño, G., Céspedes, J., Leal, M., Vargas, S. (2018). Prevalencia de factores de riesgo cardiovascular en escolares de un área rural y de una urbana en Colombia. Biomédica, 2018, 38(4),
545-554. doi.org/10.7705/biomedica.v38i4.4223

Carissimi, A., Adan, A., Tonetti, L., Fabbri, M., Hidalgo, M., Levandovski, R., Natale, V., Martoni, M. (2017). Physical self-efficacy is associated to body mass index in schoolchildren. J Pediatr, 93, 64 -9. doi.org/10.1016/j.jped.2016.04.011

Campo, L., Herazo, Y., García, F., Suarez, M., Méndez, O., Vásquez, F. (2017). Estilos de vida saludables de niños, niñas y adolescentes. Salud Uninorte, 33 (3), 419-428.

Castillo, E., Tornero, I., García, J. (2018). Relación entre actividad física, alimentación y familia en edad escolar. Retos, 34, 85-88.

Crespo, N., Corder, K., Marshall, S., Norman, G., Patrick, K., Sallis, J., Elder J. (2013). An examination of multilevel factors that may explain gender differences in children's physical activity. J Phys Act Health, 10(7), 982 — 92. doi.org/10.1123/ jpah.10.7.982

Chanal, J., Cheval, B., Courvoisier D, Paumier D. (2019). Developmental relations between motivation types and physical activity in elementary school children. Psychology of Sport and Exercise, 43, 233-242. doi.org/10.1016/ j.psychsport.2019.03.006.

Denton, S., Trenell, M., Plotz, T., Savory, L., Bailey, D., Kerr, C. (2013. Cardiorespiratory fitness is associated with hard and light intensity physical activity but not time spent sedentary in 10-14 year old schoolchildren: the HAPPY study. PLoS ONE, 8(4), e61073. doi.org/10.1371/journal.pone.0061073.

De Lepeleere, S., De Bourdeaudhuij, I., Cardon, G., Verloigne, M. (2017). The effect of an online video intervention 'Movie Models' on specific parenting practices and parental selfefficacy related to children's physical activity, screen-time and healthy diet: a quasi experimental study. BMC Public Health, 17(1), 366. doi: 10.1186/s12889-017-4264-1.

Erdim, L., Ergun, A., Kuðuoðlu, S. (2019). Reliability and validity of the Turkish version of the Physical Activity Questionnaire for Older Children (PAQ-C). Turk J Med Sci, 49, 162-169. doi:10.3906/sag-1806-212.

Fairclough. S., Ridgers, N., Welk, G. (2012). Correlates of children's moderate and vigorous physical activity during weekdays and weekends. J Phys Act Health, 9(1), 129-37. DOI: 10.1123 / jpah.9.1.129

Fradejas, E., Espada, M. (2018). Evaluación de la motivación en adolescentes que practican deportes en edad escolar. Retos, 33, 27-33.

González, M., Meléndez,A. (2013). Sedentarism, active lifestyle and sport: impact on health and obesity prevention. Nutr Hosp, 28 (5), 89-98. DOI:10.3305/nh.2013.28.sup5.6923

González, I., Martner, R. (2013). Superando el «Síndrome del casillero vacío». Determinantes de la distribución del ingreso en América Latina. Revista CEPAL, 108, 7-25.

González, S., Castiblanco, M., Arias, L., Martínez, A., Cohen, D., Holguin, G., Almanza, A., Camargo, D., Correa, J., Escobar, I., García, J., Gámez, R., Garzón, M., Herazo, Y., Hurtado, H., Lozano, O., Páez, D., Ramírez, R., Ruiz, N., Tovar, G., Sarmiento, O. (2016). Results From Colombia's 2016 Report Card on Physical Activity for Children and Youth. Journal of Physical Activity and Health, 13(2), 129-136. doi.org/10.1123/ jpah.2016-0369.

Guillamón, A., Carrillo, P., García, E., Pérez, J., Tarraga, L., Tarraga, P. (2019). Mediterranean diet, weight status and physical activity in schoolchildren of the Region of Murcia. Clínica e Investigación en Arteriosclerosis, 31(1), 1-7. doi.org/10.1016/ j.arteri.2018.09.002

Hnatiuk, J., Salmon, J., Hinkley, T., Okely, A., Trost, S. (2014). A review of preschool children's physical activity and sedentary time using objective measures. Am J Prev Med, 47(4), 487-97. 
doi.org/10.1016/j.amepre.2014.05.042

Hallal, P., Andersen, L., Bull, F., Guthold, R., Haskell, W., Ekelund, U. (2012). Global physical activity levels: surveillance progress, pitfalls, and prospects. Lancet, 380 (9838), 247-57. doi: 10.1016/S0140-6736 (12) 60646-1.

Herazo, Y., Domínguez, R. (2012). Confiabilidad del cuestionario de actividad física en niños colombianos. Rev. Sal Publ, 14 (5), 802-809.

Hernández, J., Velázquez, R., Martínez, M., Garoz, I., Tejero, C. (2011). Escala de Autoeficacia Motriz: propiedades psicométricas y resultados de su aplicación a la población escolar española. Revista de Psicología del Deporte, 20(1), 1328.

Hui-ping Sit, C., Jie Yu, J., Heung-sang Wong, S., Capio, C., Masters, R. (2019). A school-based physical activity intervention for children with developmental coordination disorder: A randomized controlled trial. Research in Developmental Disabilities, 89, 1-9. doi.org/10.1016/j.ridd.2019.03.004.

Instituto Colombiano de Bienestar Familiar, Ministerio de Protección Social, PROFAMILIA. Encuesta Nacional de la Situación Nutricional en Colombia. 2010. Disponible en: http:// www.bogotamasactiva.gov. co/?q=node/481 ENSIN2010.

Isa, T., Sawa, R., Torizawa, K., Murata, S., Saito, T., Ebina, A., Kondo, Y., Tsuboi, Y., Fukuta, A., Misu, S., Ono, R. (2019). Reliability and Validity of the Japanese Version of the Physical Activity Questionnaire for Older Children. Clin Med Insights Pediatr, 13, 1-6. doi: 10.1177/ 1179556519835833.

Jáuregui, E., López, J., Macías, A., Porras, S., Reynaga, P., Morales, J., Cabrera, J. (2013). Autoeficacia y actividad física en niños mexicanos con obesidad y sobrepeso. Revista Euroamericana de Ciencias del Deporte, 2(2), 69-75.

Janssen, I., LeBlanc, A. (2010). Systematic review of the health benefits of physical activity and fitness in school-aged children and youth. Int J Behav Nutr Phys Act, 7(40), 1-16. doi.org/ 10.1186/1479-5868-7-40.

Kitzman, H., Wilson, D., Van Horn, M., Lawman, H. (2010). Relationship of body mass index and psychosocial factors on physical activity in underserved adolescent boys and girls. Health Psychol, 29(5), 506 - 13. Doi: 10.1037/a0020853.

Manchola, J., Bagur, C., Girabent, M. (2017). Fiabilidad de la versión española del cuestionario de actividad física PAQ-C/ Reliability Spanish Version of Questionnaire of Physical Activity PAQ-C. Revista Internacional de Medicina y Ciencias de la Actividad Física y el Deporte, 17(65), 139-152. doi.org/ 10.15366/rimcafd2017.65.008

Martínez, A., Mayorga, D., Viciana, J. (2018). Factores predictores de la actividad física en escolares españoles de acuerdo a su estado de peso. 2018, Retos, 33, 74-80.

Méndez, A., Cecchini, J., Fernández, J. (2017). The effect of a selfconstructed material on children's physical activity during recess. Rev Saude Publica, 51(58), 1-7. doi: 10.1590 / S15188787.2017051006659.

Manley, D., Cowan, P., Graff, C., Perlow, M., Rice, P., Richey, P., Sanchez,Z. (2014). Self-Efficacy, Physical Activity, and Aerobic Fitness in Middle School Children: Examination of a Pedometer Intervention Program. Journal of Pediatric Nursing, 29(3), 228 237. doi.org/10.1016/j.pedn.2013.10.011

Moore, J., Hanes, J., Barbeau, P., Gutin, B., Treviño, R., Yin, Z. (2007). Validation of the Physical Activity Questionnaire for Older Children in children of different races. Pediatr Exerc Sci, 19(1), 6-19.

Núñez, J., Zurita, F., Ramírez, I., Lozano, A., Puertas, P., Ubago, J. (2019). Análisis de la relación entre los hábitos físico-saludables y la dieta con la obesidad en escolares de tercer ciclo de
Primaria de la Provincia de Granada. Retos, 35, 31-35.

Olaya, P., Bastidas, M., Arvidsson, D. (2015). Los niños colombianos con sobrepeso y obesidad necesitan apoyo motivacional adicional en la escuela para realizar una actividad física que mejore su salud. Diario de la actividad física y la salud, 12(5), 604-609. doi.org/10.1123/jpah.2014-0024

Palomino, C., Reyes, F., Sánchez, A. (2018). Niveles de actividad física, calidad de vida relacionada con la salud, autoconcepto físico e índice de masa corporal: un estudio en escolares colombianos. Biomédica, 38, 224-31. doi.org/10.7705/ biomedica.v38i0.3964

Piñeros, M., Pardo, C. (2010). Actividad física en adolescentes de cinco ciudades colombianas: resultados de la Encuesta mundial de Salud a escolares. Rev Sal Pub, 12(6), 903-914.

Prieto, D., Correa, J., Ramírez, R. (2015). Niveles de actividad física, condición física y tiempo en pantallas en escolares de Bogotá, Colombia: estudio FUPRECOL. Nutr. Hosp, 32(5), 2184 - 2192. doi.org/10.3305/nh.2015.32.5.9576.

Popeska, B., Jovanova, S., Chin, M., Edginton, C., Mok, M., Gontarev, S. (2018). Implementation of brain breaks $₫$ in the classroom and effects on attitudes toward physical activity in a macedonian school setting. Int J Environ Res Public Health, 15(6), 1-18. doi:10.3390/ijerph15061127.

Ríos, Y., Navarro, R., Arufe, V., Pérez, J. (2018). Evaluación de un programa de actividad física mediante juegos populares en escolares de Educación Primaria. Retos, 34, 108-113.

Rosa, A., Garcia, E., Carrillo, P. (2019). Relación entre capacidad aeróbica y el nivel de atención en escolares de primaria. Retos, 35, 36-41.

Quan, M., Zhang, H., Zhang, J., Zhou, T., Zhang, J., Zhao, G., Fang, H., Sun, S., Wang, R., Chen, P. (2019). Are preschool children active enough in Shanghai: an accelerometer-based crosssectional study. BMJ Open, 9(4), e024090. doi:10.1136/ bmjopen-2018-024090.

Rivera, J., González, T., Pedraza, L., Aburto, T., Sánchez, T., Martorell, R. (2013). Childhood and adolescent overweight and obesity in Latin America: a systematic review. Lancet Diabetes Endocrinol, 70(13), 173-6.

Seguin, R., Perry, C., Solanki, E., Mc Calmont, J., Ward, J., Jackson, Ch. (2019). Mujeres Fuertes y Corazones Saludables, un programa de nutrición y actividad física culturalmente adaptado para latinas rurales: hallazgos de un estudio piloto. Int. J. Environ. Res. Public Health, 16(4), 630. doi:10.3390/ijerph16040630.

Sallis, J. (1993). Epidemiology of physical activity and fitness in children and adolescents. Crit Rev Food Sci Nutr, 33, 403 408.

Suton, D., Pfeiffer, K., Feltz, D., Yee, K., Eisenmann, J., Carlson, J. (2013). Physical activity and self-efficacy in normal and over-fat children. Am J Health Behav, 37(5), 635-40. DOI: 10.5993/AJHB.37.5.

Vidarte, J., Vélez, C., Parra, J. (2019). Physical activity and body mass index in Colombian schoolchildren. Multicenter study. Hacia promoc. Salud, 24(1), 44-55. DOI: 10.17151/ hpsal.2019.24.1.5.

Zurita, F., Castro, M., Rodríguez, S., Cofré, C., Chacón, R., Martínez, A., Muros, J. (2017). Actividad física, obesidad y autoestima en escolares chilenos: Análisis mediante ecuaciones estructurales. Rev. Méd. Chile, 145(3), 299-308. doi.org/ 10.4067/S0034-98872017000300006. 